\title{
Information Sharing, Resource Integrating and Decision Optimizing: Study on Emergency Management Mode from the Perspective of Synergetics
}

\author{
Zeng Yuhang ${ }^{1}$,2, Wang Jinhua ${ }^{1}$, Xu Xiaodong' \\ ${ }^{1}$ School of Public Administration, Huazhong University of Science and Technology, Wuhan, \\ P.R.China, 430074 \\ ${ }^{2}$ School of Public Administration, Guizhou University of Finance and Economics, Guiyang, \\ P.R.China, 550004 \\ (E-mail:yuhangzeng@qq.com, xiaodong-xu@mail.hust.edu.cn, lovewangjinhua@yahoo.com)
}

\begin{abstract}
Emergency Management is facing the challenge of the frequent occurrences and chain reaction of disasters. There are some problems in the traditional emergency management, such as the collaborations with multi-agents emergency management, neglect of precaution, lack of mechanism of resource integration, so it is very hard for traditional emergency management mode to implement multi-agents and cross-sectoral management. This paper applies Synergetic principles to analyze the order parameters of emergency management system, and argues that emergency management synergy mode can be established from three aspects: information sharing, resource integrating and organization decision making optimized.
\end{abstract}

Key words: Emergency; Emergency Management; Information Sharing; Resource Integrating; Decision Making Optimizing; Synergy Management

\section{Introduction}

With the continuous advance of China's urbanization and industrialization, the society has entered a high time when the emergencies happen frequently. Influenced by contradictions and conflicts, such as various natural disasters, social and psychological imbalances, economic interests to pursue and other, emergencies are characteristic of complexity and network. The reasons that an emergency happens and develops partly are diverse predisposing factors and the emergency concurrently causes other crises and disasters. Once the emergency happened, the effects and hazards will develop swiftly and the consequence is maximized because of their interdependent relationship, thus presenting a complicated situation. Ulrich Beck believes that in the risk society, two features, the growing proliferation of human uncertainty logic and the trend of social structure towards a complex, random and split state make the consequence more diverse and complex, so that the traditional methods of risk 
control and tools become dwarf and out of control. With this situation, the traditional emergency management faces an unprecedented challenge. China for many years still takes the traditional management mode which includes gradation, classification, and the principle of territorial management. In addition, emergency resources and teams are scattered in various departments in which mutual cooperation and collaboration more depend on the temporary administrative coordination from the superiors in the face of cross-sectoral , inter-regional and comprehensive response to the invasion of the disaster chain and disaster networks, thus making the rescue inefficient and wasteful of resources. With the continuous development of information technology, collaborative government theory and practice become mature, and the mechanism of emergency management on the e-government platform has been established. In order to explore China's emergency management mode, this paper, from the Perspective of Synergetics, not only analyses the emergencies and its impacts, but also points out the defects of each city in establishing the current emergency response mechanism. Furthermore, this paper discusses how to establish a multi-agents and trans-department collaborative mode.

\section{Emergency Chain Reaction and Impacts}

Many studies have shown that diffusion, chain and mass are the characters of emergency in addition to the serious results and urgent rescue. Dombrowsky holds the harms of emergency itself is limited, but the consequences will trigger a chain reaction, resulting in a more serious crisis and disasters. Understanding of the knock-on effect and its mechanism of emergencies, plays a very important role in the control of disasters and the reduction of losses. The analysis of chain reaction can take event chain mode, social physics and catastrophe mode and other methods.

A large number of cases show that diversity of vulnerable factors and inducing factors in human society and the nature often make one-cause-to-more-re -sults and more-causes-to-one-result. The development of emergences is not isolated, which changes the social and natural environment, and the interaction between emergencies makes them integrative, thus causing the consequences out of control. Recently, ice and snow disasters frequently occur in China south, making the traffic disruptive, coal and power short, telecommu- nication interruptive, and other difficulties concerning people's livelihood and social instability. The American scholars take the interdependence between the city infrastructure and the collapse of city function as "waterfall collapse", the "upgrade crash" and "the collapse of the common incentive".

Because of the interdependence, the emergencies form a chain structure, reflecting the complex relationship of reciprocal causation between them. Inspired by the character of the chain events, we should first analyze these complex factors, and establish a monitoring mechanism of information in a timely manner to block one of the predisposing factors, which can effectively prevent the occurrence of unexpected events. The evolution of the chain events show their mobility and transformation, which increase the difficulty of monitoring and responding to emergencies, so a department has been far from being able to effectively capture and analyze information on the chain events and it 
must depend on collaborative networks to effectively deal with.

\section{The Structural Defects of Emergency Linkage Response Mechanism}

In recent years, along with the deepening understanding of the disaster and its chain reaction, the academic circles and the emergency managers gradually realize the importance of multi-agents and trans-department collaboration, and then begin to integrate the various emergency resources in order to set up the coordinated emergency mechanism. Multi-agents collaborative emergency exploration in China, began with the emergency linkage response mechanism that means when the emergencies come, the emergent administrative departments should obey the unified command and coordination in agreement with the regulations and rules, then this joint action makes the response to the variety of disasters more quick, thus realizing a unified dispatch in face of the public emergencies within a certain district.

The emergency linkage response mechanism has pooled the information about the social security, traffic accidents, medical emergency and even the mayor hot line, and refers to a good way to serve the citizens and the emergent agents. However, when disasters and various derivative disasters occur, emergency linkage mechanism is only a stimulus-response system, which transmits information in a unidirectional way. And the present administrative departments divided by classification and gradation take actions in their own way that is different between departments, thus hindering the effective prevention and disposal in face of emergencies and chain disasters. This paper concludes the following defects that the emergency linkage mechanism itself has:

(1) The management agent defects: unable to coordinate the actions from multi-agents

Emergent linkage mechanism shows its advantages in the regular accidents concerning water, power, gas, cars, social security and other unexpected events. But this traditional emergent management is unable to deal with catastrophes, especially whose scope is far beyond the local governments and professional sectors, because this management is exclusive to emergent information, emergent resource, emergent decision and execution to coordinate the different governments or sectors, so that it is difficult to get rescue from more emergent agents.

(2) Functional defects: too much emphasis on afterwards emergent management.

At present, the operation mode of the emergency linkage mechanism mainly emphasizes on-duty emergent management, in other words, a process in the dealing phrase and is blind to the daily management and data in the phrase of prevention and monitor. When the chain disasters come, the emergent linkage system should not only provide" the past" and" current" data, also show " future" disaster development trend, the expected consequences, interventional measures, emergent decision, the assessment of expected rescue outcome , and all-round monitoring. These functions demand all levels of governments closely connective with other departments to keep cooperation, and sharing information .

(3) Resource integration defects: lack of emergency resources integration mechanism.

The emergency linkage mechanism is composed of the resources scattered in different departments, which are dispatched and deployed only by the temporary 
administrative coordination. In the current system, the emergency supplies, the rescue team, emergency sites and emergency funds are not distributed effectively, dispatched reasonable, disposed properly, then isolation and dispersion are characteristic of those resources. Accordingly, on one hand, the resources cannot be mobilized easily, on the other, duplication of resources may still be constructed.

\section{From Linkage to Synergism: Integrated Emergency Management Mode}

Faced with the emergent disasters, the traditional emergency management mode which based on the emergency response mechanism needs to be changed. Western scholars and emergency management agencies had established a theoretical foundation for the collaborative mode of emergency management between government departments and NGO collaborative study over the years. Haken's synergetic theory, studied the self-organization system dominated by the universal law, broadened the vision of the emergency management in collaborative research, and analyses the characteristics of the emergency management system from Synergetics perspective, which help to grasp the collaborative nature of emergency management and the general law. It provides a theoretical tool for the analysis of emergency management collaborative mode.

Synergetics argues, the macroscopic properties of a nonlinear disordered activity in the complex open systems are reflected by the competition and cooperative relations between the order parameters. When an order parameter subsystem generates self-organization orderly, and this subsystem , through its coordination, dominates other subsystems to produce the same self-organized activities, the whole system functions in the same pattern, making it from imbalance to balance.

From the synergetic theory, the impact of order parameter on the macro-elements in the emergency management system shows that the nature of emergency management agents, mainly referring to the capabilities, reflects the various characteristics of the order parameter which promote the emergency management system organized orderly. According to the study on organizational core competency, key factors to affect the Government's emergency management capabilities, include emergency information, emergency resources protection and emergency response organizations and execution, which comprise driving force for the emergency management coordination system to achieve a synergistic effect. Therefore, emergency management collaborative mode should include these three aspects: to achieve information sharing between multi-agents; to integrate and optimize resources scattered in various emergency agents; and finally to make collaborative decision-making and effective execution .

\subsection{Basis of Synergism: Emergency Information Sharing}

Emergency information is the basis for emergency management, and the whole process of emergencies' evolvement, is inseparable from the emergency information management. The process of emergency evolvement, from its beginning to development and changes, need emergency managers continuously making decision under the changing circumstances, which should be 
based on the variety of information the emergencies showed. The mechanism of formation of the aforementioned incident analysis found that the occurrence of unexpected events and a large number of influencing factors, easily develop in a intangible way, and out of control from the manager. Emergency management must be established based not only on timely monitoring and control of emergencies, but also on the collection and processing of all kinds of information to determine the state of socio-economic imbalance in the system, integrate various resources to take appropriate measures to deal with timely blocking relevant factors, to improve the socio-economic security of the system. To cope with the challenges of the disaster chain of unexpected events, the agency at the center of emergency information management mode falls far short of the requirements of information collaboration. Traditional information processing mode is a relatively simple mode, from which the various departments are divided according to the simple division of labor, then information can only flow up and down between the level of each sector that is the lack of effective communication and feedback, and the emergency management system is decentralized to each other and isolation, shown in Figure 1.

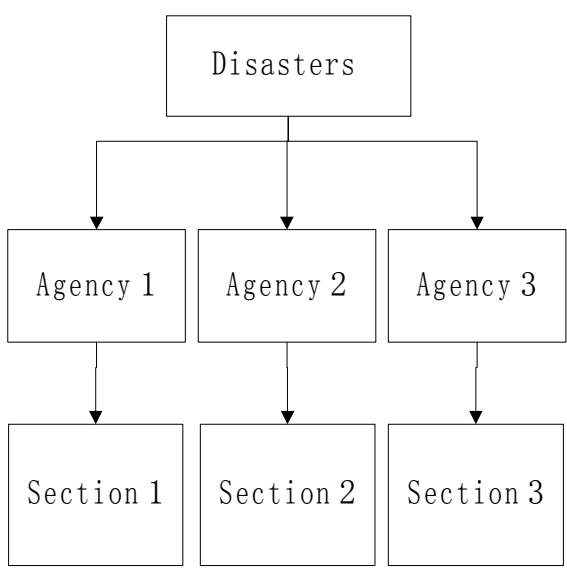

a. Traditional Mode

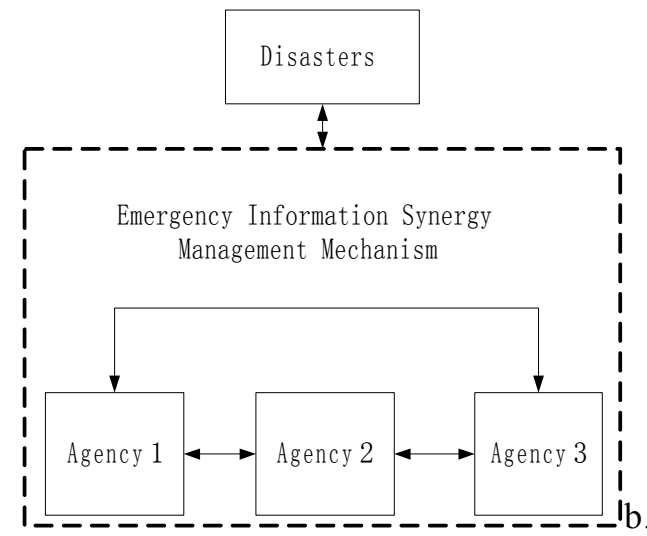

Emergency Information Synergy

Management Mode

Fig1. Comparison of Emergency Information Management Mode

What should we do for the information sharing? This paper holds that information synergy mechanism established by an emergent information data center is a good way, instead of rebuilding a comprehensive emergency management organization, adjusting the existing administrative system, re-constructing the relationship between the various departments. Difficulties of emergency information sharing source are, apart from institutional factors, mainly the information technology infrastructure that lagged behind the fragmentation which brought about backward compatibility issues, and information management tools and other 
problems are also the reasons. Solving these problems, a unified data center should be constructed, where emergency information standardization system and synergy platform based on SOA architecture must be created, thus forming a synergy mode of emergency information, a high degree of information sharing between emergency response agencies. Under emergency information synergy management mode, the various departments, from simple access to reporting or receiving incident information into the full monitoring, tracking, emergency information, and real-time data exchange and sharing with other professionals and emergency response agencies, can take timely prevention and control measures to overcome the emergency linkage mechanism that only passively receives information and emergency measures of which emphasize afterwards emergency management instead of prevention.

\subsection{Synergetic Means : Emergency \\ Resource Integration}

In emergency management, emergency resources and support systems play crucial roles, and among emergency resources include man resources, material and other, such as emergency rescue teams, consulting team, emergency funds, emergency supplies, shelter, emergency telecommunications and etc. Once an emergency occurs, the emergency rescue system needs allocate the resources for the emergency management, not only to ensure adequate resources, but also achieve rapid and timely supply in place. To solve the problem of emergency support system, we should overcome two difficulties: First, to pool the emergent resources and promote the sharing of information between departments; second to coss the boundaries between departments, to change the current division and the sub-sector system, and based on hazard and vulnerability analysis to take the collaborative construction of the emergency management system as an important part. Emergency resources synergy management mechanism should provide enough information to support the demand analysis and make the emergency scientific and standard.First, through the analysis of inducing-disaster factors and vulnerability, the need of emergency resource becomes salient and its integrity lead to collaborative work, which is called formation mechanism; Secondly, on the collaborative platform, the various agents of emergency resources and support system fully realize that the information exchange and collaborative work can achieve the optimal allocation of emergency resources, which is the implementation mechanism; Finally, the organization through collaboration to improve the efficiency of emergency management objectives, results in a further collaborative momentum, which is the driving force mechanism. These three mechanisms can achieve the interaction between organizations, not only reaching the goal of synergy, but ensuring that the whole system functions smoothly and effectively.

\subsection{Goals of Synergism: Emergency Decision Optimization}

Emergency response agencies on the basis of information resources and support collaborative mechanism ensure that emergent departments at all levels achieve collaboration in decision making and the implementation, and its essence is the Organization's action synergism. In decision-making level, emergency management agency's base it on the information coordination, according to the 
emergencies at all stages of the dynamic information to constantly adjust their decision-making objectives, then the overall effect of the optimal individual decision is made under the domination of the global target, achieving decision-making synergy. When implementation of collaborative organizations in the information, resources and decision-making synergies turns to the executive implementation, collaboration effect will reach. Important means of achieving the coordination in the decision making and execution is to establish an emergency management platform, which is ensured by the information and resource coordination to protect the cooperative system, provide a unified Command and Control platform. This emergency management has three important functions: First, the central command system for emergency decision-making is the entire emergency management system controller, responsible for the control and coordination; Decision implementation monitoring and control systems ensure the emergency decisions effectively implemented; it plays an important role in decision making, decision standardized and scientific, thus enhancing the ability of information processing and response.

\section{Conclusions}

With the changes of social, economic, natural and political environment, all kinds of emergencies occur frequently, so governments should carry out multi-agents and cross-sectoral synergetic emergency management, which aims to cope with a chain reaction which is a big challenge when facing the chain disasters. However, the traditional sector division for emergency management and its backwards become major factors in restricting the government collaboration and timely responding to emergencies, thereby affecting the governments to achieve prevention, reduction of harms, rapid response, and coordinated response made by emergency management. From the collaborative perspective , government emergency management system is an open, non-linear and non-equilibrium complex system, which is in line with the law of the work from cooperative system. Based on the analysis of the collaborative order parameter, this paper argues that emergency management collaborative mode should include the following three aspects: emergency information sharing, emergency guarantee system, emergency synergy between organizations. Only give full play to the role of collaborative mechanism, can the emergency management activities in various types and at different levels form a collaborative system to challenge the continuously growing network of disasters.

\section{References}

[1] DOMBROWSKY W. R. Again and again: is a disaster what we call Disaster? Some conceptual notes on conceptualizing the object of disaster sociology[J]. International Journal of Mass Emergencies and Disasters, 2003(13):241-254.

[2] Zeng Jian etc, Social SynergiticsWuhan: Science Press, 2000(in Chinese)

[3] Drabek T E, Hoetmer G J, Emergency Management: Principles and Practice for Local Government. Washington, D.C.: International City Management Association, 1991. 
[4] H.Haken, Synergetics[M].Shanghai: Shanghai Translation Press, 2006(in Chinese)

[5] William L. Waugh, Jr. , Terrorism, Homeland Security and the National Emergency Management Network, Public Organization Review NO.3, 2003.

[6] Wu Guobin etc, Empirical Research on Factors that affected efficiency of Multi-Agent Emergency Management Synergy.Research on Engineering. 2011 3(2):166(in Chinese) 